\title{
APPPLICATIONS OF RADON-NIKODÝM THEOREMS TO MARTINGALE CONVERGENCE
}

\author{
BY
}

J. J. UHL, JR.

Introduction. The subject of convergence of martingales of functions with values in a Banach space was first treated by Scalora [17] and Chatterji [3] who independently showed that a martingale of functions with values in a reflexive Banach space obeys roughly the same basic convergence theorems as martingales of real or complex valued functions. Various authors have continued the work; among them being A. and C. Ionescu-Tulcea [9], who generalized the earlier work by considering martingale convergence theorems from the point of view of abstract ergodic theory; Chatterji [4], who generalized his earlier work by making certain assumptions on the range space of the martingale of functions; and Métivier [14] and [15] who has treated in detail not only the earlier problems but also related problems dealing with both weak and strong martingales and martingales with values in locally convex linear topological spaces.

In one of his papers [15, Theorem 11] Métivier proved a theorem characterizing those Banach space valued measures which admit a representation as an indefinite Bochner integral with respect to a finite nonnegative real valued measure. More recently, Rieffel [16] gave a different, but related, characterization of such vector valued measures. Since the connection between the theory of derivations and the theory of convergence of martingales is well established, a natural problem is to use the above mentioned Radon-Nikodým theorems of Métivier and Rieffel to obtain a characterization of norm convergent Banach valued martingales and other results. The purpose of this paper is to do exactly this.

The first section is concerned with preliminaries which establish the setting for the work which follows. The second section, which is the main section of the paper, is devoted to characterization of norm convergent martingales in certain Orlicz spaces $L^{\Phi}$ of vector valued functions. The third section continues with an extension of theorems of Doob, Krickeberg and Chatterji dealing with measure convergence and almost everywhere convergence of $L^{1}(\mathfrak{X})$-bounded martingales. Finally, in $\S 4$, the Radon-Nikodým-Bochner theorem for finitely additive measures is generalized to the vector valued case.

1. Some preliminaries. Throughout this paper, unless otherwise noted, $(\Omega, \Sigma, \mu)$ is a fixed finite measure space; i.e. $\mu$ is a countably additive nonnegative real valued

Received by the editors February 27, 1969. 
finite set function defined on a $\sigma$-field $\Sigma$ of subsets of the point set $\Omega$. $\mathfrak{X}$ is a Banach space, $\Phi$ is a Young's function [22] with complementary function $\Psi . L^{\Phi}(\Omega, \Sigma, \mu, \mathfrak{X})$ $\left(=L^{\Phi}(\mu, \mathfrak{X})\right)$ is the collection of all totally $\mu$-measurable [6, III.2.10] $\mathfrak{X}$-valued functions $f$ which satisfy $\int_{\Omega} \Phi(\|f\| / k) d \mu<\infty$ for some $k>0$. Upon the introduction of the equivalent norms

$$
N_{\Phi}(f)=\inf \left\{k>0: \int_{\Omega} \Phi(\|f\| / k) d \mu \leqq 1\right\}
$$

or

$$
\|f\|_{\Phi}=\sup \left\{\int_{\Omega}\|f\| g d \mu: g>0 \quad \int_{\Omega} \Psi(g) d \mu \leqq 1\right\}
$$

$[10, \mathrm{pp} .19,22]$ and the identification of functions which differ on at most a $\mu$-null set, $L^{\Phi}(\mu, \mathfrak{X})$ becomes a Banach space. If there exists a constant $k$ such that $\Phi(2 x) \leqq$ $k \Phi(x)$ for all real $x, \Phi$ is said to obey the $\Delta_{2}$-condition. $L^{1} \supset L^{\Phi}$, and all $L^{p}$ spaces $1 \leqq p<\infty$ are found among the $L^{\Phi}$ spaces with $\Phi$ obeying the $\Delta_{2}$-condition. When $\Phi$ obeys the $\Delta_{2}$-condition, simple functions are dense in $L^{\Phi}(\mu, \mathfrak{X})$. In addition

LEMMA 1. Let $\mathscr{F}$ be a subfield of $\Sigma$ such that the o-field generated by $\mathscr{F}$ is $\Sigma$. If $\Phi$ obeys the $\Delta_{2}$-condition, then the linear span of the set

$$
\left\{x \chi_{E}: x \in \mathfrak{X}, E \in \mathscr{F}\right\}
$$

is dense in $L^{\Phi}(\mu, \mathfrak{X})$. (Here $\chi_{E}(\omega)=+1, \omega \in E ; \chi_{E}(\omega)=0, \omega \notin E$.)

The proof, which will be omitted, follows quickly from the facts that simple functions are dense in $L^{\Phi}(\mu, \mathfrak{X})$, and that $\lim _{\mu(E) \rightarrow 0} N_{\Phi}\left(x \chi_{E}\right)=0$ for all $x \in \mathfrak{X}$, and that for $E \in \Sigma$, there exists [6, III.71] $\left\{E_{n}\right\} \subset \mathscr{F}$ such that $\lim _{n \rightarrow \infty} \mu\left(E_{n} \triangle E\right)=0$ where $E_{n} \triangle E=\left(E_{n}-E\right) \cup\left(E-E_{n}\right)$.

Definition 1. Let $f \in L^{1}(\mu, \mathfrak{X})$ and $B$ be a sub- $\sigma$-field of $\Sigma$. A function $g \in L^{1}(\mu, \mathfrak{X})$ is the conditional expectation of $f$ given $B$, if $g$ is $B$-measurable and $\int_{E} f d \mu=\int_{E} g d \mu$ for all $E \in B$. In this case one writes $E^{B}(f)=g$. Here, as throughout, the integral is that of [6, Chapter III].

The operator $E^{B}$ is defined for all $f \in L^{1}(\mu, \mathfrak{X})$ and when considered as an operator $E^{B}: L^{\Phi}(\mu, \mathfrak{X}) \rightarrow L^{\Phi}(\mu, \mathfrak{X})$ is a linear contraction.

DEFINITION 2. Let $T$ be a directed set and $\left\{B_{\tau}, \tau \in T\right\}$ be an increasing net of sub- $\sigma$-fields of $\Sigma$; i.e. $\tau_{1} \leqq \tau_{2}$ implies $B_{\tau_{1}} \subset B_{\tau_{2}} .\left\{f_{\tau}, B_{\tau}, \tau \in T\right\}$ is a martingale in $L^{\Phi}(\mu, \mathfrak{X})$ if each $f_{\tau} \in L^{\Phi}(\mu, \mathfrak{X})$ and $\tau_{1} \leqq \tau_{2}$ implies $E^{B_{\tau_{1}}}\left(f_{\tau_{2}}\right)=f_{\tau_{1}}$.

This section will be terminated with a lemma which will be employed crucially in the next section. It is only a slight modification of a well known result.

Lemma 2. Let $\Phi$ obey the $\Delta_{2}$-condition. A martingale $\left\{f_{\tau}, B_{\imath}, \tau \in T\right\}$ converges in the $L^{\Phi}$ norm if and only if there exists a function $f \in L^{\Phi}(\mu, \mathfrak{X})$ such that

for all $E \in \bigcup_{\tau} B_{\tau}$.

$$
\lim _{\tau} \int_{E} f_{\tau} d \mu=\int_{E} f d \mu
$$


Proof (Sufficiency). Since $\left\{B_{\imath}, \tau \in T\right\}$ is an increasing net of $\sigma$-fields, it follows easily that $\bigcup_{\tau} B_{\tau}$ is a subfield of $\Sigma$. By standard techniques, without loss of generality it will be assumed that the $\sigma$-field generated by $\bigcup_{\tau} B_{\tau}$ is $\Sigma$. Now let $\varepsilon>0$ be given. By Lemma 1, there exists a function $f_{\varepsilon}=\sum_{i=1}^{n} x_{i} \chi_{E_{i}}: x_{i} \in \mathfrak{X}, E_{i} \in \bigcup_{\tau} B_{\tau}$, such that $N_{\Phi}\left(f-f_{\varepsilon}\right)<\varepsilon / 2$. Since $\left\{B_{\tau}, \tau \in T\right\}$ is an increasing net, there exists a $\tau_{0} \in T$ such that for all $\tau \geqq \tau_{0}\left\{E_{i}\right\}_{i=1}^{n} \subset B_{\tau}$. Hence for $\tau \geqq \tau_{0} E^{B_{\tau}}\left(f_{\varepsilon}\right)=f_{\varepsilon}$. Moreover for $\tau \geqq \tau_{1}, \int_{E} f_{\tau} d \mu=\int_{E} f_{\tau_{1}} d \mu$ for $E \in B_{\tau_{1}}$ by the martingale property. It follows that $E^{B_{\tau}}(f)=f_{\tau}$.

Therefore for $\tau \geqq \tau_{0}$,

$$
N_{\Phi}\left(f-f_{\tau}\right) \leqq N_{\Phi}\left(f-f_{\varepsilon}\right)+N_{\Phi}\left(f_{\varepsilon}-f_{\tau}\right)=N_{\Phi}\left(f-f_{\varepsilon}\right)+N_{\Phi}\left(E^{B_{\tau}}\left(f_{\varepsilon}-f\right)\right) \leqq 2 N_{\Phi}\left(f-f_{\varepsilon}\right) \text {, }
$$

since $E^{B_{\tau}}$ is a contraction, $<2 \varepsilon / 2=\varepsilon$, by the choice of $f_{\varepsilon}$. This establishes the sufficiency of the condition.

To prove the necessity, suppose $\lim _{\tau} f_{\tau}=f$ in $N_{\Phi}$ norm. Then since the operation defined for $g \in L^{\Phi}(\mu, \mathfrak{X})$ by $g \rightarrow \int_{E} g d \mu$, is a bounded linear operator for each $E \in \Sigma$, it follows that

$$
\lim _{\tau} \int_{E} f_{\tau} d \mu=\int_{E} f d \mu
$$

for all $E \in \bigcup_{\tau} B_{\tau}$. This proves the necessity and the lemma.

2. The mean convergence of Banach valued martingales. This section is devoted to characterizing those martingales $\left\{f_{\tau}, B_{\imath}, \tau \in T\right\} \subset L^{\Phi}(\mu, \mathfrak{X})$ such that

$$
\lim _{\tau} N_{\Phi}\left(f_{\tau}-f\right)=0
$$

for some $f \in L^{\Phi}(\mu, \mathfrak{X})$ when $\Phi$ obeys the $\Delta_{2}$-condition. Some results along these lines have been obtained by Chatterji [3] and [4], Scalora [17], A. and C. IonescuTulcea [9], and Métivier [14] and [15]. Although the conditions given in Theorem 2 below bear some relation to those of Métivier [15, Theorem 4, p. 190] it is believed that Theorem 2 is the first theorem which provides a necessary and sufficient condition for a martingale to converge in the mean. The proof of that theorem hinges on the following proposition which is basic to all of the considerations of this paper. Essentially, it translates the Radon-Nikodým theorems of Métivier [15, Theorem 11] and Rieffel [16] into a form usable in the present context.

(In the statement of Proposition 1 below, the wording “....weakly (norm) compact..." refers to two separate conditions. The proposition is true if read "... a weakly compact..." and it is equally true if read "... a norm compact...". Thus Proposition 1 is actually two separate propositions. This wording is employed in the interest of economy of space and will be used in other statements in this paper.)

Proposition 1. Let $\Sigma_{0}$ be subfield of $\Sigma$ such that $\Sigma$ is the $\sigma$-field generated by $\Sigma_{0}$. 
Let $\mu$ be a finite nonnegative measure on $\Sigma$. If $F$ is a countably additive $\mathfrak{X}$-valued measure defined on $\Sigma_{0}$, then $F$ admits the representation

$$
F(E)=\int_{E} f d \mu, \quad E \in \Sigma_{0}
$$

for some $f \in L^{1}(\mu, \mathfrak{X})$ if and only if

(i) $F$ is of bounded variation;

(ii) $F$ is $\mu$-continuous; $i$.

$$
\lim _{\mu(E) \rightarrow 0}\|F(E)\|=0
$$

and

(iii) Given $\varepsilon>0$ there exists a weakly (norm) compact convex subset $K \subset \mathfrak{X}$ such that for any $\delta>0$ there exists $E_{0}\left(=E_{0}(\varepsilon, K, \delta)\right) \in \Sigma_{0}$ with $\mu\left(\Omega-E_{0}\right)<\varepsilon$ and $F(E) \in \mu(E) K+\delta U$ for all $E \subset E_{0}, E \in \Sigma_{0}$, where $U$ is the closed unit ball $\{\|x\| \leqq 1\}$ of $\mathfrak{X}$.

Proof (Sufficiency). Let $F$ satisfy (i), (ii), and (iii). The argument of [19, p. 39] shows that since $F$ is $\mu$-continuous, $F$ has a $\mu$-continuous (hence countably additive) extension (still denoted by $F$ ) of bounded variation to $\Sigma$. Now let $\varepsilon>0$ be given and by (iii) choose a convex weakly (norm) compact set $K$ and sets $\left\{E_{n}\right\} \subset \Sigma_{0}$, $\mu\left(\Omega-E_{n}\right)<\varepsilon$ such that

$$
F(E) \in \mu(E) K+n^{-2} U
$$

for $E \subset E_{n}, E \in \Sigma_{0}, n=1,2, \ldots$ Now consider $E_{0}=\lim \sup _{n} E_{n}=\bigcap_{n=1}^{\infty} \bigcup_{m=n}^{\infty} E_{m}$. By standard results of measure theory [8]

$$
\mu(\Omega)-\varepsilon \leqq \lim _{n} \sup \mu\left(E_{n}\right) \leqq \mu\left(E_{0}\right) .
$$

That is, $\mu\left(\Omega-E_{0}\right)<\varepsilon$. Also if $E \subset E_{0}, E \in \Sigma$, then $E \subset \bigcup_{m=n}^{\infty} E_{m}$ for each $n$. Hence if $n$ is fixed and $S_{n}=E_{n}, S_{n+1}=E_{n+1}-E_{n}, \ldots, S_{n+k+1}=E_{n+k+1}-\left(\bigcup_{m=n}^{n+k} E_{n}\right), \ldots$ then the sequence $\left\{S_{m}\right\}_{m=n}^{\infty}$ is disjoint and $E \subset \bigcup_{m=n}^{\infty} S_{m}$. Moreover since $F$ on $\Sigma$ is countably additive,

$$
F(E)=F\left(E \cap\left(\bigcup_{m=n}^{\infty} S_{m}\right)\right)=\sum_{m=n}^{\infty} F\left(E \cap S_{n}\right)
$$

Next note that $E \cap S_{m} \subset S_{m} \subset E_{m}$ for all $m \geqq n$. Therefore, according to [6, III. 7.1], there exists for each $m$, a sequence $\left\{H_{m j}\right\} \subset \Sigma_{0}$, which may be assumed to satisfy $\left\{H_{m j}\right\} \subset E_{m}$, such that $\lim _{j} \mu\left(\left(E \cap S_{m}\right) \triangle H_{m j}\right)=0$. The $\mu$-continuity of $F$ and the identities

$$
\mu\left(G_{1} \triangle G_{2}\right)=\mu\left(G_{1}-G_{2}\right)+\mu\left(G_{2}-G_{1}\right)
$$

and

$$
F\left(G_{1}\right)-F\left(G_{2}\right)=F\left(G_{1}-G_{2}\right)-F\left(G_{2}-G_{1}\right)
$$


guarantee that $\lim _{j} F\left(H_{m j}\right)=F\left(E \cap S_{m}\right)$ strongly in $\mathfrak{X}$, and $\lim _{j} \mu\left(H_{m j}\right)=\mu\left(E \cap S_{m}\right)$, $m \geqq n$. Since $H_{m j} \subset E_{m}, x_{m j} \in K$ and $y_{m j} \in U$ can be chosen such that

$$
F\left(H_{m j}\right)=\mu\left(H_{m j}\right) x_{m j}+\frac{y_{m, j}}{m^{2}}, \quad m \geqq n, j \geqq 1 .
$$

Since $K$ is weakly (norm) compact, the Eberlein-Smulian theorem guarantees that it can (and will) be assumed without loss of generality that $\lim _{j} x_{n j}=x_{n} \in K$ exists in the weak topology of $\mathfrak{X}$. But then for $m \geqq n$

$$
\begin{aligned}
\left\|F\left(E \cap S_{m}\right)-\mu\left(E \cap S_{m}\right) x_{m}\right\| & \leqq \liminf _{j}\left\|F\left(H_{m j}\right)-\mu\left(H_{m j}\right) x_{m j}\right\| \\
& \leqq \lim _{j} \sup \left\|m^{-2} y_{m j}\right\| \leqq m^{-2} .
\end{aligned}
$$

Hence for each $m \geqq n$, one can write

$$
F\left(E \cap S_{m}\right)=\mu\left(E \cap S_{m}\right) W_{m}+m^{-2} Z_{n}
$$

where $W_{m} \in K$ and $Z_{m} \in U$. Then

$$
\begin{aligned}
F(E)=\sum_{m=n}^{\infty} F\left(E \cap S_{m}\right) & =\sum_{m=n}^{\infty}\left(\mu\left(E \cap S_{m}\right) W_{m}+m^{-2} Z_{m}\right) \\
& =\sum_{m=n}^{\infty} \mu\left(E \cap S_{m}\right) W_{m}+\sum_{m=n}^{\infty} m^{-2} Z_{m},
\end{aligned}
$$

since both series are absolutely convergent. Rearranging yields

$$
\left\|F(E)-\sum_{m=n}^{\infty} \mu\left(E \cap S_{m}\right) W_{m}\right\|=\left\|\sum_{m=n}^{\infty} m^{-2} Z_{m}\right\| \leqq \sum_{m=n}^{\infty} m^{-2} .
$$

Now, note that

$$
\sum_{m=n}^{\infty} \mu\left(E \cap S_{m}\right) W_{m}=\mu(E) \sum_{m=n}^{\infty} \frac{\mu\left(E \cap S_{m}\right)}{\mu(E)} W_{m} \quad(0 / 0=0) .
$$

But since $K$ is convex and closed, and $\sum_{m=n}^{\infty} \mu\left(E \cap S_{m}\right) / \mu(E)=1$,

Moreover,

$$
K_{n}=\sum_{m=n}^{\infty} \frac{\mu\left(E \cap S_{n}\right)}{\mu(E)} W_{m} \in K
$$

$$
0 \leqq \lim _{n \rightarrow \infty}\left\|F(E)-\mu(E) K_{n}\right\| \leqq \lim _{n \rightarrow \infty} \sum_{m=n}^{\infty} m^{-2}=0 .
$$

It follows that $F(E) \in \mu(E) K$ for all $E \subset E_{0}, E \in \Sigma$. This shows that in the case $K$ is weakly compact that $F$ satisfies the hypothesis of the Radon-Nikodým theorem of Métivier [15, Theorem 11, p. 203], and in the case $K$ is norm compact $F$ satisfies the Radon-Nikodým theorem of Rieffel [16, p. 466]. In any case there exists $f \in L^{1}(\mu, \mathfrak{X})$ such that

$$
F(E)=\int_{E} f d \mu, \quad E \in \Sigma
$$

and in particular for $E \in \Sigma_{0}$. This completes the proof of the sufficiency. 
(Necessity). Suppose there exists $f \in L^{1}(\mu, \mathfrak{X})$ such that

$$
F(E)=\int_{E} f d \mu, \quad E \in \Sigma_{0} .
$$

Then $F$ has an obvious "natural" extension to $\Sigma$, still denoted by $F$. Standard properties of the integral show that $F$ is $\mu$-continuous. Furthermore, it is well known that the variation of $F$ is just $\int_{\Omega}\|f\| d \mu<\infty$. Hence (i) and (ii) are satisfied. Now let $\varepsilon>0$ be given. According to the Radon-Nikodým theorem of Rieffel [16, Proposition 1.12 , p. 472 ] there exists a set $E_{0} \in \Sigma, \mu\left(\Omega-E_{0}\right)<\varepsilon / 2$ and a norm compact (hence weakly compact) set $K$, which by Mazur's theorem may be assumed convex, such that $F(E) \in \mu(E) K, E \subset E_{0}, E \in \Sigma$. Next, let $\delta>0$ be given and by [6, III.7.1], choose $E_{\delta} \in \Sigma_{0}$ subject to $\mu\left(E_{0} \triangle E_{\delta}\right)<\beta$ where $\beta=\min \{\varepsilon / 2, \delta / 2 m, \alpha\}$ and $m-1=\max \{\|x\|: x \in K\}$, and $\mu(E)<\infty, \alpha>0$, implies $\|F(E)\|<\delta / 2$. Then $\mu(\Omega-E) \leqq \mu\left(\Omega-E_{0}\right)+\mu\left(E_{0} \triangle E_{\delta}\right)<\varepsilon / 2+\varepsilon / 2=\varepsilon$. Now let $E \subset E_{\delta}, E \in \Sigma_{0}$. By hypothesis there exists $x \in K$ such that $F\left(E \cap E_{0}\right)=\mu\left(E \cap E_{0}\right) x$. For this $x$, one has

$$
\begin{aligned}
\|F(E)-\mu(E) x\| & \leqq\left\|F(E)-F\left(E \cap E_{0}\right)\right\|+\left\|\mu\left(E \cap E_{0}\right) x-\mu(E) x\right\| \\
& \leqq\left\|F\left(E-E_{0}\right)\right\|+\|x\| \mu\left(E-E_{0}\right)<\delta / 2+\delta / 2
\end{aligned}
$$

by the choice of $\beta$ and the fact that $E-E_{0} \subset E_{\delta}-E_{0}$. This proves the necessity of the condition and the theorem.

The following result, which characterizes mean convergent martingales in certain $L^{\Phi}(\mu, \mathfrak{X})$ spaces is the main result of this paper.

THEOREM 2. Let $\Phi$ obey the $\Delta_{2}$-condition and let $\left\{f_{\tau}, B_{\tau}, \tau \in T\right\}$ be a martingale in $L^{\Phi}(\mu, \mathfrak{X})$. The net $\left\{f_{\tau}, \tau \in T\right\}$ is convergent in the $L^{\Phi}(\mu, \mathfrak{X})$ norm if and only if

(i) there exists a constant $m<\infty$ such that $\sup _{\tau \in T} N_{\Phi}\left(f_{\tau}\right) \leqq m$;

(ii) $\left\{f_{\tau}, \tau \in T\right\}$ is terminally uniformly integrable; i.e. given $\varepsilon>0$ there is a $\delta>0$ and an index $\tau_{0} \in T$ such that $\mu(E)<\delta$ implies $\int_{E}\left\|f_{\tau}\right\| d \mu<\varepsilon, \tau \geqq \tau_{0}$; and

(iii) given $\varepsilon>0$ there exists a weakly (norm) compact convex set $K \subset \mathfrak{X}$ such that for any $\delta>0$ there is an index $\tau_{0} \in T$ and a set $E_{0} \in B_{\tau_{0}}, \mu\left(\Omega-E_{0}\right)<\varepsilon$ satisfying $\tau \geqq \tau_{0}$ implies

$$
\int_{E} f_{\tau} d \mu \in \mu(E) K+\delta U
$$

for all $E \subset E_{0}, E \in B_{\tau}$.

In the case that $\Psi$, the Young's function complementary to $\Phi$, satisfies $\Psi(x)<\infty$ for $x<\infty$ (ii) above is implied by (i) and may be dropped.

Proof. Without loss of generality, it will be assumed throughout the entire proof that $\Sigma$ is generated by $\bigcup_{\tau} B_{\tau}$.

(Necessity). Suppose that $\lim _{\tau} f_{\tau}=f$ in the $L^{\Phi}$ topology. Then $\lim _{\tau} N_{\Phi}\left(f_{\tau}\right)$ $=N_{\Phi}(f)$. On the other hand since $E^{B}$ is a contraction, the fact that $\left\{f_{\tau}, B_{\tau}, \tau \in T\right\}$ is a martingale ensures that $\left\{N_{\Phi}\left(f_{\tau}\right), \tau \in T\right\}$ is an increasing net. Therefore $N_{\Phi}\left(f_{\tau}\right) \leqq$ 
$N_{\Phi}(f)$. This proves (i). To prove (ii), note that $L^{\Phi}$ convergence implies $L^{1}$ convergence. Hence

$$
\lim _{\tau}\left|\int_{E}\left\|f_{\tau}\right\| d \mu-\int_{E}\|f\| d \mu\right| \leqq \lim _{\tau} \int_{E}\left\|f_{\tau}-f\right\| d \mu \leqq \lim \left\|f_{\tau}-f\right\|_{1}=0
$$

uniformly in $E \in \Sigma$. (ii) follows immediately from the $\mu$-continuity of the indefinite integral $\int_{(\cdot)}\|f\| d \mu$. To prove (iii), let $\varepsilon>0$ be given. By Proposition 1 , there is a weakly (norm) compact convex subset $K \subset \mathfrak{X}$ such that given any $\delta>0$ there is an $E_{\delta} \in \bigcup_{\tau} B_{\tau}$ such that

$$
\int_{E} f d \mu \in \mu(E) K+\delta U
$$

$E \subset E_{\delta}, E \in \bigcup_{\tau} B_{\tau}$. For a fixed $\delta>0$ select $\tau_{0} \in T$ such that $E_{\delta} \in B_{\tau_{0}}$. Then for $\tau_{0} \leqq \tau$ and $E \in B_{\tau}, E \subset E_{\delta}$, one has, by the martingale property

$$
\int_{E} f_{\tau} d \mu=\int_{E} f d \mu \in \mu(E) K+\delta U
$$

This proves the necessity.

(Sufficiency). The sufficiency will be proven first for $L^{1}(\mu, \mathfrak{X})$. Define the set function $F$ on $\bigcup_{\tau} B_{\tau}$ by

$$
F(E)=\lim _{\tau} \int_{E} f_{\tau} d \mu, \quad E \in \bigcup_{\tau} B_{\tau} .
$$

The martingale property ensures that the defining net on the right is eventually constant for $E \in \bigcup_{\tau} B_{\tau}$, and hence $F$ is well defined and obviously finitely additive. From (i), for any finite disjoint collection $\left\{E_{n}\right\}_{n=1}^{m} \subset \bigcup_{\tau} B_{\tau}$,

$$
\begin{aligned}
\sum_{n=1}^{m}\left\|F\left(E_{n}\right)\right\| & =\sum_{n=1}^{m}\left\|\lim _{\tau} \int_{E_{n}} f_{\tau} d \mu\right\| \\
& =\sum_{n=1}^{m}\left\|\int_{E_{n}} f_{\tau_{0}} d \mu\right\| \leqq \int_{\Omega}\left\|f_{\tau_{0}}\right\| d \mu \leqq M
\end{aligned}
$$

for $\tau_{0} \in T$ chosen such that $\left\{E_{n}\right\}_{n=1}^{m} \subset B_{\tau_{0}}$. Hence $F$ is of bounded variation. (ii) clearly implies the $\mu$-continuity, and therefore the countable additivity of $F$.

To complete the proof of the sufficiency in the $L^{1}(\mu, \mathfrak{X})$ case, by Lemma 2 , it need only be shown that there exists $f \in L^{1}(\mu, \mathfrak{X})$ such that

$$
F(E)=\lim _{\tau} \int_{E} f_{\tau} d \mu=\int_{E} f d \mu, \quad E \in \bigcup_{\tau} B_{\tau} .
$$

This is clearly the same as showing $F$ has a Radon-Nikodým derivative. Let $\varepsilon>0$ be given and let $K$ be as in (iii). By (iii) for any fixed choice of $\delta>0$ there exists an index $\tau_{0} \in T$ and $E_{0} \in B_{\tau_{0}}, \mu\left(\Omega-E_{0}\right)<\varepsilon$ such that $\int_{E} f_{\tau} d \mu \in \mu(E) K+\delta U$ for $E \subset E_{0}$, 
$E \in B_{\imath}, \tau \geqq \tau_{0}$. So, let $E \subset E_{0}, E \in \bigcup_{\iota} B_{\tau}$ be arbitrary, then $E \in B_{\tau_{0}}$ for some $\tau_{1} \geqq \tau_{0}$ and

$$
F(E)=\int_{E} f_{\tau_{1}} d \mu \in \mu(E) K+\delta U
$$

Hence, by Proposition 1 , there exists $f \in L^{1}(\mu, \mathfrak{X})$ such that

$$
F(E)=\int_{E} f d \mu=\lim _{\tau} \int_{E} f_{\tau} d \mu
$$

for $E \in \bigcup_{\imath} B_{\imath}$. This, combined with Lemma 2 completes the proof in the $L^{1}(\mu, \mathfrak{X})$ case.

Moving to the general case, assume (i), (ii) and (iii) hold. Since $\mu(\Omega)<\infty, N_{\Phi}$ dominates the $L^{1}(\mu, \mathfrak{X})$ norm, and the martingale converges in $L^{1}(\mu, \mathfrak{X})$, by the above, to a function $f \in L^{1}(\mu, \mathfrak{X})$. Clearly $f$ satisfies

$$
\lim _{\tau} \int_{E} f_{\tau} d \mu=\int_{E} f d \mu \quad \text { for } E \in \bigcup_{\tau} B_{\imath} .
$$

Since $\left\{f_{\imath}, \tau \in T\right\}$ converges to $f$ in $L^{1}(\mu, \mathfrak{X})$ norm, there exists a sequence of indices $\left\{\tau_{n}\right\} \subset T$ such that $\lim _{n}\left\|f_{\tau_{n}}-f\right\|_{1}=0$ and such that $\lim _{n} f_{\tau_{n}}(\omega)=f(\omega)$ a.e. But since $N_{\Phi}\left(f_{\tau_{n}}\right) \leqq M$

$$
\int_{\Omega} \Phi\left(\left\|f_{\tau_{n}}\right\| /(M+1)\right) d \mu \leqq 1 \quad \text { for all } n
$$

and by Fatou's lemma,

$$
\int_{\Omega} \Phi(\|f\| /(M+1)) d \mu \leqq \lim _{n} \inf \int_{\Omega} \Phi\left(\left\|f_{\tau_{n}}\right\| /(M+1)\right) d \mu \leqq 1
$$

Hence $f \in L^{\Phi}(\mu, \mathfrak{X})$, and since $\lim _{\tau} \int_{E} f_{\tau} d \mu=\int_{E} f d \mu, E \in \bigcup_{\tau} B_{\tau}$, an application of Lemma 2 concludes the proof of the sufficiency.

To complete the proof of the theorem, suppose $\Psi$, the Young's function complementary to $\Phi$ satisfies $\Psi(x)<\infty$ for $x<\infty$. Assuming (i), one has

$$
\int_{E}\left\|f_{\tau}\right\| d \mu=\int_{\Omega}\left\|f_{\tau}\right\| \chi_{E} d \mu \leqq N_{\odot}\left(f_{\tau}\right)\left\|\chi_{E}\right\|_{\Psi} \leqq M\left\|\chi_{E}\right\|_{\Psi}
$$

But $\lim _{\mu(E) \rightarrow 0}\left\|\chi_{E}\right\|_{\Psi}=0$, provided $\Psi(x)<\infty$ for $x<\infty$. Hence the net $\left\{f_{\tau}, \tau \in T\right\}$ is uniformly integrable and $a$ fortiori satisfies (ii). Q.E.D.

Specializing Theorem 2 to the case where the index set $T$ is $N$, the positive integers with the natural order, is the following theorem.

THEOREM 3. Let $\left\{f_{n}, B_{n}\right\}$ be a martingale satisfying the hypothesis of Theorem 2. If $f_{\infty}$ is the $L^{1}$-limit of $\left\{f_{n}\right\}$, then $\lim _{n \rightarrow \infty} f_{n}(\omega)=f_{\infty}(\omega)$ for $\mu$-almost all $\omega \in \Omega$. 
Proof. This is a consequence of a theorem [9, p. 189] due to A. and C. IonescuTulcea which asserts that a mean convergent martingale converges almost everywhere to its $L^{1}$-limit. Q.E.D.

Theorem 3 can be weakened somewhat. This is the topic of $\S 3$.

3. $L^{1}(\mu, \mathfrak{X})$-bounded martingales. This section is concerned with supplying sufficient conditions for an $L^{1}(\mu, \mathfrak{X})$-bounded martingale to converge in measure and almost everywhere. The classical result in this direction is due first to Doob and later to Krickeberg who proved that if $\left\{f_{\tau}, B_{\tau}, \tau \in T\right\}$ is a martingale of real valued functions with $\left\|f_{\tau}\right\|_{1} \leqq M<\infty$ then the net $\left\{f_{\tau}, \tau \in T\right\}$ converges in measure to a function $f \in L^{1}(\mu)$ and if $T=N$, the positive integers, then this convergence is also $\mu$-almost everywhere. Recently, in the case $T=N$, Chatterji [4, Theorem 2] generalized this to $\mathfrak{X}$-valued functions by placing certain convenient assumptions on the Banach space $\mathfrak{X}$. In this section results are obtained by placing assumptions on the martingale itself. It will be shown that the results here subsume all of the above mentioned results.

THEOREM 4. Let $\left\{f_{\tau}, B_{\imath}, \tau \in T\right\}$ be a martingale in $L^{1}(\mu, \mathfrak{X})$ satisfying

(i) $\sup _{\tau} \int_{\Omega}\left\|f_{\tau}\right\| d \mu \leqq M \leqq \infty$; and

(ii) for each $\varepsilon>0$ there exists a weakly compact convex set $K$ such that given $\delta>0$ there is an index $\tau_{0} \in T$ and $E_{0} \in B_{\tau_{0}}, \mu\left(\Omega-E_{0}\right)<\varepsilon$ such that for $\tau \geqq \tau_{0}$

$$
\int_{E} f_{\tau} d \mu \in \mu(E) K+\delta U
$$

for all $E \subset E_{0}, E \in B_{\imath}$, then there exists $f \in L^{1}(\mu, \mathfrak{X})$ such that $\lim _{\tau} f_{\tau}=f$ in $\mu$-measure. If, in addition, $T=N$, then $\lim _{n \rightarrow \infty} f_{n}(\omega)=f(\omega)$, strongly in $\mathfrak{X}$, a.e. $[\mu]$ as well.

Proof. As in the proof of Theorem 2, define $F$ on the field $\bigcup_{\tau} B_{\tau}$ by

$$
F(E)=\lim _{\tau} \int_{E} f_{\tau} d \mu, \quad E \in \bigcup_{\tau} B_{\tau} .
$$

Then, as in the proof of Theorem 2, $F$ is finitely additive, and (i) implies that $F$ is of bounded variation. Following standard methods [4, p. 57], one can write $F=G+H$, where $G$ and $H$ are $\mathfrak{X}$-valued finitely additive set functions of bounded variation where $|G|$, the variation of $G$ is singular with respect to $\mu$ (i.e. given $\varepsilon>0, \delta>0$ there exists $\left.E \in \bigcup_{\tau} B_{\tau}, \mu(E)<\varepsilon,|G|(\Omega-E)<\delta\right)$, and $H$ is a countably additive set function which is $\mu$-continuous. First it will be shown that there is an $h \in L^{1}(\mu, \mathfrak{X})$ such that $H(E)=\int_{E} h d \mu, E \in \bigcup_{\tau} B_{\tau}$.

For this, note that $H$ satisfies (i) and (ii) of the hypothesis of Proposition 1. To show that $H$ satisfies (iii) of that hypothesis, let $\varepsilon>0$ be given and choose a weakly compact convex set $K$ such that for any $\delta>0$ there is an index $\tau_{0} \in T$ and $E_{0} \in B_{\imath}, \mu\left(\Omega-E_{0}\right)<\varepsilon / 2$ such that $\tau \geqq \tau_{0}$ implies

$$
\int_{E} f_{\tau} d \mu \in \mu(E) K+(\delta / 2) U
$$


$E \subset E_{0}, E \in B_{t}$. Now let $\delta>0$ be fixed. Choose the corresponding index $\tau_{0} \in T$ and set $E_{0} \in B_{\tau_{0}}$ as above; also choose $\tilde{E} \in \bigcup_{\tau} B_{\tau}$ such that $\mu(\widetilde{E})<\varepsilon / 2$ and $|G|(\Omega-\widetilde{E})$ $<\delta / 2$. Setting $E_{1}=E_{0} \cap(\Omega-\widetilde{E})$, and choosing $\tau_{1} \geqq \tau_{0}$ such that $E_{1} \in B_{\tau_{0}}$ one has $\mu\left(\Omega-E_{1}\right)<\varepsilon$ and for $\tau \geqq \tau_{1}$

$$
\int_{E} f_{\imath} d \mu \in \mu(E) K+(\delta / 2) U,
$$

$E \in B_{\imath}, E \subset E_{1} \subset E_{0}$. But for $E \in B_{\imath}, F(E)=\int_{E} f_{\imath} d \mu$, by the martingale property. Hence

$$
F(E) \in \mu(E) K+(\delta / 2) U,
$$

$E \subset E_{1}$. But $\|H(E)-F(E)\| \leqq\|G(E)\| \leqq|G|\left(F_{1}\right) \leqq|G|(\Omega-\tilde{E})<\delta / 2, E \subset E_{1}, E \in \bigcup_{\imath} B_{\imath}$. Hence

$$
H(E) \in \mu(E) K+\delta U .
$$

This shows $H$ satisfies (iii) of Proposition 1 and therefore establishes the existence of $h \in L^{1}(\mu, \mathfrak{X})$ such that

$$
H(E)=\int_{E} h d \mu, \quad E \in \bigcup_{\tau} B_{\tau} .
$$

Next, define $h_{\tau}$ by $h_{\tau}=E^{B_{\tau}(h)}$ and note that for $E \in B_{\tau}, F(E)=\int_{E} f_{\tau} d \mu$. Hence for $E \in B_{\tau}, G(E)=\int_{E}\left(f_{\tau}-h_{\tau}\right) d \mu$. Since $f_{\tau}-h_{\tau}$ is $B_{\tau}$-measurable there exists a $B_{\tau^{-}}$ measurable $g_{\imath}\left(=\left(f_{\tau}-h_{\imath}\right)\right)$ such that $G(E)=\int_{E} g_{\tau} d \mu, E \in B_{\imath}$. Also since $\left\{f_{\imath}, B_{\imath}, \tau \in T\right\}$ and $\left\{h_{\imath}, B_{\imath}, \tau \in T\right\}$ are martingales, so is $\left\{g_{\imath}, B_{\imath}, \tau \in T\right\}$. Moreover, $\left\{h_{\imath}, B_{\imath}, \tau \in T\right\}$ is an $L^{1}$-convergent martingale by Lemma 2 since $\lim _{\tau} \int_{E} h_{\tau} d \mu=\int_{E} h d \mu, E \in B_{\tau}$. Since this martingale is convergent in $L^{1}(\mu, \mathfrak{X})$, it is also convergent in $\mu$-measure to $h_{0} \in L^{1}(\mu, \mathfrak{X})$.

Finally, it will be shown that $\lim _{\tau} g_{\tau}=0$ in $\mu$-measure. For this, let $0<\varepsilon<1$ and $\delta>0$ be given. Since $|G|$ and $\mu$ are singular, there exists $E \in \bigcup_{\tau} B_{\tau}$ such that

$$
\mu(\Omega-E)+|G|(E)<\delta \varepsilon / 2 .
$$

Now choose an index $\tau_{0} \in T$ such that $E \in B_{\tau_{0}}$. Then for any $\tau \geqq \tau_{0}$

$$
\begin{aligned}
\mu\left(\left\{\omega:\left\|g_{\tau}(\omega)\right\|>\varepsilon\right\}\right) & =\mu\left(\left\{\omega \notin E:\left\|g_{\tau}(\omega)\right\|>\varepsilon\right\}\right)+\mu\left(\left\{\omega \in E:\left\|g_{\tau}(\omega)\right\|>\varepsilon\right\}\right) \\
& \leqq \mu(\Omega-E)+(1 / \varepsilon) \int_{E \cap\left\{\omega:\left\|g_{\tau}(\omega)\right\|>\varepsilon\right\}}\left\|g_{\imath}\right\| d \mu \\
& \leqq \mu(\Omega-E)+(1 / \varepsilon) \int_{E}\left\|g_{\imath}\right\| d \mu \\
& \leqq \mu(\Omega-E)+(1 / \varepsilon)|G|(E),
\end{aligned}
$$

since $\int_{E}\left\|g_{\tau}\right\| d \mu \leqq|G|(E)<\delta \varepsilon / 2+(1 / \varepsilon) \delta \varepsilon / 2<\delta$, by the choice of $E$ and $\varepsilon$. This implies $\lim _{\tau} g_{\tau}=0$ in $\mu$-measure and that $\lim _{\tau}\left(h_{\tau}+g_{\tau}\right)=h_{0} \in L^{1}(\mu, \mathfrak{X})$ in $\mu$-measure. This completes the proof of the first statement. 
To finish the proof of the theorem, let $T=N$, form the martingales $\left\{f_{n}, B_{n}\right\}$, $\left\{g_{n}, B_{n}\right\}$ and $\left\{h_{n}, B_{n}\right\}, f_{n}=g_{n}+h_{n}$ as above. As noted above, $\lim _{n \rightarrow \infty} h_{n}=h_{0}$ exists strongly in $L^{1}(\mu, \mathfrak{X})$. Therefore, a theorem of A. and C. Ionescu-Tulcea and Neveu [15, p. 189], [9, p. 117], $\lim _{n} h_{n}(\omega)=h_{0}(\omega)$ strongly in $\mathfrak{X}$ for $\mu$-almost all $\omega \in \Omega$. On the other hand, $\left\{\left\|g_{n}\right\|, B_{n}\right\}$ is an $L^{1}$-bounded submartingale which converges to zero in measure and therefore almost everywhere. Hence $\lim _{n} f_{n}(\omega)=h_{0}(\omega)$ strongly in $\mathfrak{X}$ for $\mu$-almost all $\omega \in \Omega$. Q.E.D.

It is not immediately obvious that this theorem does, in fact, contain the result of Chatterji [4, Theorem 2] as advertised. To proceed in this direction, a definition is needed. A Banach space $\mathfrak{X}$ has the Radon-Nikodým property with respect to $\mu$ if every $\mathfrak{X}$-valued $\mu$-continuous countably additive set function of bounded variation defined on $\Sigma$ admits the representation

$$
F(E)=\int_{E} f d \mu, \quad E \in \Sigma
$$

for some $f \in L^{1}(\mu, \mathfrak{X})$. All reflexive spaces and separable dual spaces have the Radon-Nikodým property with respect to an arbitrary finite measure $\mu$.

The next result shows (ii) of the hypothesis of Theorem 4 is redundant in the case that $\mathfrak{X}$ has the Radon-Nikodým property with respect to $\mu$.

THEOREM 5. Let $\mathfrak{X}$ have the Radon-Nikodým property with respect to $\mu$. Then any martingale $\left\{f_{\tau}, B_{\tau}, \tau \in T\right\}$ with

$$
\sup _{\tau}\left\|f_{\tau}\right\|_{1} \leqq M<\infty
$$

has the property that given an $\varepsilon>0$ there exists a norm (hence weakly) compact convex subset $K \subset \mathfrak{X}$ such that for any choice of $\delta>0$ there is an index $\tau_{0} \in T$ and $E_{0} \in B_{\tau_{0}}$ with $\mu\left(\Omega-E_{0}\right)<\varepsilon$ such that for $\tau \geqq \tau_{0}$

$$
\int_{E} f_{\tau} d \mu \in \mu(E) K+\delta U
$$

for $E \subset E_{0}, E \in B_{\imath}$.

Proof. As in the proof of Theorem 4, write $\lim _{\tau} \int_{E} f_{\tau} d \mu=F(E)=G(E)+H(E)$, $E \in \bigcup_{\tau} B_{\tau}$, where by the current hypothesis, $F$ is of bounded variation and so are $G$ and $H . H$ is $\mu$-continuous and countably additive, while $G$ is singular with respect to $\mu$. Now, as in [19, p. 39] $H$ has a $\mu$-continuous countably additive extension of bounded variation to $\sigma\left(\bigcup_{\tau} B_{\tau}\right)$. Therefore since $\mathfrak{X}$ has the Radon-Nikodým property with respect to $\mu$ there exists $h \in L^{1}(\mu, \mathfrak{X})$ such that $H(E)=\int_{E} h d \mu$ for all $E \in \bigcup_{\tau} B_{\tau}$. Now let $\varepsilon>0$ be given. According to Proposition 1, there exists a norm compact convex set $K$ such that for any fixed $\delta>0$ there is $E^{\prime}\left(=E^{\prime}(\delta, K)\right) \in \bigcup_{\tau} B_{\tau}$ with $\mu\left(\Omega-E^{\prime}\right)<\varepsilon / 2$ such that

$$
H(E) \in \mu(E) K+(\delta / 2) U
$$

Also since $G$ is $\mu$-singular, there exists a set $E^{\prime \prime} \in \bigcup_{\tau} B_{\tau}$ with $\mu\left(\Omega-E^{\prime \prime}\right)<\varepsilon / 2$ and 
$|G|\left(E^{\prime \prime}\right)<\delta / 2$. ( $|G|$ is the variation of $G$.) Setting $E_{0}=E^{\prime} \cap E^{\prime \prime}$, one finds easily that $\mu\left(\Omega-E_{0}\right)<\varepsilon$ and $F(E) \in \mu(E) K+\delta U$ for $E \subset E_{0}, E \in \bigcup_{\tau} B_{\tau}$. But if $\tau_{0} \in T$ is chosen such that $E_{0} \in B_{\tau_{0}}$ then

$$
\int_{E} f_{\tau} d \mu=F(E) \in \mu(E) K+\delta U
$$

for $\tau \geqq \tau_{0}$ and $E \subset E_{0}, E \in B_{\imath}$. This completes the proof of the theorem.

An immediate consequence of Theorems 4 and 5 is

Corollary 6 (Chatteru [4, Theorem 2]). If $\mathfrak{X}$ satisfies the Radon-Nikodým property with respect to $\mu$ then any martingale indexed by $N,\left\{f_{n}, B_{n}\right\}$ with

$$
\sup _{n \geqq 1} \int_{\Omega}\left\|f_{n}\right\| d \mu \leqq M<\infty
$$

converges almost everywhere.

Theorem 5 can also be used to deduce the theorems of Scalora [17], Chatterji [3] and Ionescu-Tulcea [9] on mean convergence directly from the sufficiency part of Theorem 2.

4. A generalization of the Radon-Nikodým-Bochner theorem. Although the Radon-Nikodým theorem fails when the assumption of countable additivity is weakened to finite additivity, Bochner [1], [6, IV. 9.14] proved the following theorem.

THEOREM (BOCHNER). Let $\mathscr{F}$ be a field of subsets of a point set $\Omega$. Let $\mu$ be a nonnegative finite finitely additive set function defined on $\mathscr{F}$. If $F$ is a real valued, finitely additive function defined on $\mathscr{F}$, of bounded variation and $\dot{\mu}$-continuous, then for each $\varepsilon>0$, there is a $\mu$-integrable simple function $f_{\varepsilon}$ such that the function $F_{\varepsilon}$ defined by the equation $F_{\varepsilon}(E)=\int_{E} f_{\varepsilon} d \mu, E \in \mathscr{F}$ satisfies the inequality $\left|F-F_{\varepsilon}\right|(\Omega)<\varepsilon$.

This theorem has been treated at length in the literature [2], [13], and [7]. In [19, Theorem III. 3] the author generalized this theorem to include $\mathfrak{X}$-valued functions for reflexive Banach spaces $\mathfrak{X}$. The purpose of this section is to use Proposition 1 to characterize, for general Banach spaces $\mathfrak{X}$, the $\mathfrak{X}$-valued finitely additive set functions which allow such an approximation. The integral is that of $[6$, Chapter III].

THEOREM 6. Let $\mathscr{F}$ be a field of subsets of a point set $\Omega$. Let $\mu$ be a nonnegative finite finitely additive set function defined on $\mathfrak{X}$. Let $F$ be a finitely additive $\mathfrak{X}$-valued set function defined on $\mathscr{F}$. Then for each $\varepsilon>0$ there exists an $\mathfrak{X}$-valued $\mu$-integrable $f_{\varepsilon}$ such that the function $F_{\varepsilon}$ defined on $\mathscr{F}$ by the equation $F_{\varepsilon}(E)=\int_{E} f_{\varepsilon} d \mu, E \in \mathscr{F}$, satisfies $\left|F-F_{\varepsilon}\right|(\Omega)<\varepsilon$ if and only if

(i) $F$ is of bounded variation;

(ii) $F$ is $\mu$-continuous, and 
(iii) given $\varepsilon>0$ there exists a weakly (norm) compact convex subset $K \subset \mathfrak{X}$ such that for any $\delta>0$ there exists $E_{\delta} \in \mathscr{F}, \mu\left(\Omega-E_{\delta}\right)<\varepsilon$ such that $F(E) \in \mu(E) K+\delta U$ for $E \subset E_{\delta}, E \in \mathscr{F}$.

Proof (Sufficiency). By [6, IV. 9.10-11] there exists a totally disconnected compact Hausdorff space $S_{1}$ such that

(a) $\Sigma_{1}$, the field of all clopen subsets of $S_{1}$ is isomorphic to $\mathscr{F}$ under a Boolean isomorphism $\tau: \mathscr{F} \rightarrow \Sigma_{1}$; and

(b) every finitely additive real valued set function on $\Sigma_{1}$ is countably additive.

Define $F_{1}$ and $\mu_{1}$ on $\Sigma_{1}$ by $F_{1}(\tau(E))=F(E)$ and $\mu_{1}(\tau(E))=\mu(E)$, respectively. Now, $\mu_{1}$ is finite and has a unique extension, still denoted by $\mu_{1}$, to $\sigma\left(\Sigma_{1}\right)$, the $\sigma$-field generated by $\Sigma_{1}$. Also since $\mu_{1}$ is countably additive and $F_{1}$ is clearly $\mu_{1}$ continuous on $\Sigma_{1}, F_{1}$ is countably additive. Now, to prove the sufficiency of the stated conditions let $\varepsilon>0$ be given, and note that because of the action of the isomorphism $\tau: \mathscr{F} \rightarrow \Sigma_{1}, F_{1}$ evidently satisfies the hypothesis of Proposition 1 . Hence there exists a $\mu_{1}$-measurable function $f_{1}: S_{1} \rightarrow \mathfrak{X}$ such that

$$
F_{1}(E)=\int_{E} f_{1} d \mu_{1}, \quad E \in \Sigma .
$$

But simple functions of the form $f=\sum_{i=1}^{n} x_{i} \chi_{\tau\left(E_{i}\right)}, E_{i} \in \Sigma_{1}$ are dense in $L^{1}\left(\mu_{1}, \mathfrak{X}\right)$ by [6, III. 8.3]. Hence there exist $\left\{E_{i}\right\}_{i=1}^{n} \subset \mathscr{F}$ and $\left\{x_{i}\right\}_{i=1}^{n} \subset \mathfrak{X}$ such that the $\mu_{1}$-simple function $g_{1}=\sum_{i=1}^{n} x_{i} \chi_{\tau\left(E_{i}\right)}$ satisfies $\int_{S_{1}}\left\|f_{1}-g_{1}\right\| d \mu_{1}<\varepsilon$. Now define $f_{\varepsilon}$ on $\Omega$ by $f_{\varepsilon}=$ $\sum_{i=1}^{n} x_{i} \chi_{E_{i}}$ and $F_{\varepsilon}$ on $\mathscr{F}$ by the equation $F_{\varepsilon}(E)==\int_{E} f_{\varepsilon} d \mu, E \in \mathscr{F}$. Then clearly

$$
\begin{aligned}
\left|F-F_{\varepsilon}\right|(\Omega) & \leqq\left|F_{1}-G_{1}\right|\left(S_{1}\right) \quad \text { (actually there is equality here) } \\
& =\int_{S_{1}}\left\|f_{1}-g_{1}\right\| d \mu_{1}<\varepsilon
\end{aligned}
$$

where $G_{1}(E)=\int_{E} g_{1} d \mu_{1}, E \in \sigma\left(\Sigma_{1}\right)$. This proves the sufficiency of the stated conditions.

To prove the necessity of the conditions, note that (i) and (ii) are clear. To prove (iii), choose the $\mu$-simple functions

$$
\begin{gathered}
f_{n}=\sum_{i=1}^{m_{n}} x_{n i} \chi_{E_{n i}}, \quad x_{n i} \in \mathfrak{X}, E_{n !} \in \mathscr{F}, \\
F_{n}(E)=\int_{E} f_{n} d \mu, \quad E \in \mathscr{F}
\end{gathered}
$$

such that $\left|F-F_{n}\right|(\Omega)<1 / n$. Define $f_{n}$ on $S_{1}$ by

Since

$$
\tilde{f}_{n}=\sum_{i=1}^{m_{n}} x_{n i} \chi_{\tau\left(E_{n i}\right)}
$$

$$
\begin{aligned}
\int_{S_{1}}\left\|\tilde{f}_{n}-\tilde{f}_{m}\right\| d \mu_{1} & =\int_{\Omega}\left\|f_{n}-f_{m}\right\| d \mu \\
& \leqq\left|F_{n}-F\right|(\Omega)+\left|F-F_{m}\right|(\Omega)<1 / n+1 / m,
\end{aligned}
$$


it follows that $\tilde{f}_{n}$ is a Cauchy sequence in $L^{1}\left(\mu_{1}, \mathfrak{X}\right)$ and hence converges to a function $f^{f} \in L^{1}\left(\mu_{1}, \mathfrak{X}\right)$. Let $\varepsilon>0$ be given. By Proposition 1 , there exists a weakly (norm) compact convex set $K \subset \mathfrak{X}$ such that given $\delta>0$ there is $\tau\left(E_{\delta}\right) \in \Sigma_{1}\left(\Sigma_{1}\right.$ generates $\left.\sigma\left(\Sigma_{1}\right)\right) \mu_{1}\left(S_{1}-\tau\left(E_{\delta}\right)\right)<\varepsilon$ and

$$
\int_{\tau(E)} \tilde{f} d \mu_{1} \in \mu_{1}(\tau(E)) K+\delta U
$$

for $E \subset E_{\delta}$ (i.e. $\tau(E) \subset \tau\left(E_{\delta}\right), E \in \mathscr{F}$ ). But

$$
\int_{E} f_{n} d \mu=\int_{\tau(E)} \tilde{f}_{n} d \mu_{1}
$$

and

$$
F(E)=\lim _{n} \int_{E} f_{n} d \mu=\lim _{n} \int_{\tau(E)} \tilde{f}_{n} d \mu=\int_{\tau(E)} \tilde{f} d \mu
$$

for all $E \in \mathscr{F}$. Hence

$$
F(E)=\int_{\tau(E)} \tilde{f} d \mu_{1} \in \mu_{1}(\tau(E)) K+\delta U=\mu(E) K+\delta U
$$

for $E \subset E_{\delta}, E \in \mathscr{F}$. This proves the necessity of (iii) and the theorem. Q.E.D.

REMARK. Theorem 6 provides an explicit characterization of the $S^{1}(\mathfrak{X})$ subspaces of the $V^{1}(\mathfrak{X})$ spaces studied in [19].

\section{REFERENCES}

1. S. Bochner, Additive set functions on groups, Ann. of Math. 40 (1939), 769-799.

2. S. Bochner and R. S. Phillips, Additive set functions and vector lattices, Ann. of Math. 42 1941), 316-324.

3. S. D. Chatterji, Martingales of Banach-valued random variables, Bull. Amer. Math. Soc. 66 (1960), 395-398.

4. - Comments on the martingale convergence theorem, Symposium on Probability Methods in Analysis, Loutraki, Greece, Springer, New York, 1967.

5. J. L. Doob, Stochastic processes, Wiley, New York, 1950.

6. N. Dunford, and J. T. Schwartz, Linear operators. Part I, Interscience, New York, 1958.

7. Charles Fefferman, A Radon-Nikodým theorem for finitely additive set functions, Pacific J. Math. 23 (1967), 35-45.

8. Paul R. Halmos, Measure theory, Van Nostrand, Princeton, N. J., 1950.

9. A. Ionescu-Tulcea and C. Ionescu-Tulcea, Abstract ergodic theorems, Trans. Amer. Math. Soc. 107 (1963), 107-125.

10. M. A. Krasnosel'skiǐ and Ya. B. Rutickiǐ, Convex functions and Orlicz spaces, Problems of Contemporary Mathematics, GITTL, Moscow, 1958 and Fizmatgiz, Moscow, 1959; English transl., Noorhoff, Groningen, 1961.

11. K. Krickeberg, Convergence of martingales with a directed index set, Trans. Amer. Math. Soc. 83 (1956), 313-337.

12. K. Krickeberg and C. Pauc, Martingales et dérivation, Bull. Soc. Math. France 91 (1963), 455-543.

13. S. Leader, The theory of $L^{p}$-spaces for finitely additive set functions, Ann. of Math. (2) 58 (1953), 528-543. 
14. M. Métivier, Limites projectives de mesures. Martingales. Applications, Ann. Mat. Pura Appl. (4) 61 (1963), 225-352.

15. - Martingales à valeurs vectorielles. Applications à la dérivation des mesures vectorielles, Ann. Inst. Fourier (Grenoble) 17 (1967), no. 2, 175-208.

16. M. A. Rieffel, The Radon-Nikodým theorem for the Bochner integral, Trans. Amer. Math. Soc. 131 (1968), 466-487.

17. F. S. Scalora, Abstract martingale convergence theorems, Pacific J. Math. 11 (1961), 347-374.

18. Howard G. Tucker, A graduate course in probability, Academic Press, New York, 1967.

19. J. J. Uhl, Jr., Orlicz spaces of finitely additive set functions, Studia Math. 29 (1967), 19-58.

20. - The Radon-Nikodym theorem and the mean convergence of Banach space valued martingales, Proc. Amer. Math. Soc. 21 (1969), 139-144.

21. - Martingales of vector valued finitely additive set functions, Pacific J. Math. (to appear).

22. A. C. Zaanen, Linear analysis, North-Holland, Amsterdam, 1953.

UNIVERSITY OF ILLINOIS,

URBANA, ILLINOIS 